\title{
Die seröse Meningoenzephalitis im Kindesalter
}

\author{
Non-Purulent Meningoencephalitis in Children
}

H.-M. Straßburg

Bibliografie

DOI http://dx.doi.org/

$10.1055 / \mathrm{s}-0031-1277223$

Klin Padiatr 2011; 223: 205-206

(c) Georg Thieme Verlag KG

Stuttgart · New York

ISSN 0300-8630

Korrespondenzadresse

Prof. Dr. Hans-Michael

\section{Straßburg}

Universitäts-Kinderklinik Josef Schneiderstraße 2 97080 Würzburg

Tel.: $+49 / 931 / 20127510$

Fax: $+49 / 931 / 20127858$

strassburg@mail.uni-

wuerzburg.de
Die möglichst frühzeitige Diagnose einer eitrigen Meningitis bei Fieber und/oder akut auftretenden neurologischen Symptomen, vor allem zerebralen Anfällen, ist ein unumstößliches Paradigma der Pädiatrie. Wir wissen aber aus vielen Erhebungen der letzten Jahre, dass z.B. ein erster zerebraler Anfall bei Fieber heute nur noch sehr selten in Folge einer bakteriellen Meningitis auftritt $[8,10]$. Wir wissen auch, dass sich das Spektrum der klinischen Symptome einer Meningoenzephalitis einerseits und der diese verursachenden Erreger andererseits in den vergangenen 20 Jahren deutlich gewandelt haben $[7,9,12]$.

Heute muss bei jedem Kind mit chronischen Kopfschmerzen, Abgeschlagenheit, Wesensveränderungen, Schlafstörungen, Schwindel und vor allem Hirnnervenausfälle auch bei nur diskretem Meningismus an eine entzündliche Erkrankung der Hirnhäute und des Gehirns gedacht werden $[2-4,7]$. Darüber hinaus ist bei allen akuten schlaffen Lähmungen im Kindes- und Jugendalter eine Liquordiagnostik indiziert, um ein Guillain-Barré-Syndrom oder eine transverse Myelitis zu differenzieren, nicht zuletzt aber auch, um eine Poliovirusinfektion auszuschließen [Reinheimer $\mathrm{C}$ et al. Enterovirusdiagnostik bei aseptischer Meningitis im Kindesalter mit Liquor und/oder Stuhl: Zellkulturgestützte Virusisolierung oder PCR? In diesem Heft: S. 221-226]. Die große Bedeutung von Borrelieninfektionen bei zirkumskripten Hirnnervenausfällen und transverser Myelitis ist sehr gut bekannt [3]. Weniger bekannt ist, dass diese Symptome auch durch Mycoplasma-pneumoniae-Infektionen ausgelöst werden können [Meyer Santeur PM et al. Mycoplasma pneumoniae-associated encephalitis in childhood-nervous system disorder during or after a respiratory tract infection. In diesem Heft: S. 209-213], offensichtlich spielen diese Erreger bei zunehmend mehr Patienten mit Symptomen einer Enzephalomyelitis eine Rolle $[4,7,11,12]$. Dabei ist es noch unklar, ob die Pathophysiologie durch die direkte Infektion oder indirekte immunologische Mechanismen zu erklären ist. Auch der Zusammenhang einer ADEM-Erkrankung bzw. einer sich daraus entwickelnden Enzephalomyelitis disseminata (=multipler Sklerose) mit vorangegangenen Infektionen (z.B. durch Epstein-Barr-Virus) ist ebenso unklar wie $\mathrm{Zu}$ sammenhänge zwischen einer Devic-Erkrankungen oder einer NMDA-Rezeptor-Enzephalopathie und vorausgehenden Entzündungen [7,12]. In jedem Fall ist für diese immer größer werdende Gruppe von post- und parainfektiösen Erkrankungen die ausführliche Liquoruntersuchung mit differenzierter Bestimmung von ver- schiedenen Entzündungsparametern sowohl für die Diagnostik als auch für die Wahl der Therapie von wesentlicher Bedeutung. Noch immer ist nicht geklärt, wann Antibiotika (Cephalosporine, Makrolide u.a.), wann Kortikoide oder Immunglobuline und wann andere immunmodulierende Therapien indiziert sind $[4,7,11,12]$. Darüber hinaus sollte immer wieder daran gedacht werden, dass verschiedene maligne Erkrankungen, u.a. Leukämien und Lymphome, primär durch Hirnnervenparesen auffällig werden [2].

Leider besteht heute bei vielen Eltern und Kindern eine große Abwehr, wenn eine Lumbalpunktion zur Liquorgewinnung vorgeschlagen wird. Immer noch bestehen Vorurteile gegenüber der „Rückenmarkspunktion“, der angeblich so schmerzhaften und traumatisierenden Untersuchung, den nach der Punktion auftretenden Rücken- und Kopfschmerzen mit Übelkeit und anderen angeblich lang dauernden postpunktionellen Gesundheitsstörungen [5,6].

Um dem entgegenzuwirken, setzt sich zu Recht zunehmend durch, dass vor allem geplante Lumbalpunktionen bei Kindern und auch bei Jugendlichen heute, wenn möglich oder gewünscht, in Kurznarkose, z. B. auch mit Lachgas, durchgeführt werden. Darüber hinaus sollten möglichst dünnlumige „pencil point“-Nadeln oder Spezialnadeln wie die „Sprotte-Nadel“ verwendet werden, um eine größere Liquorleckage am Punktionsort zu vermeiden $[1,5,6]$.

Auch in der Zukunft wird bei vielen entzündlichen und nicht-entzündlichen Krankheitsbildern in der Pädiatrie eine Liquorgewinnung notwendig sein. Es ist wichtig, dass diese möglichst wenig traumatisierend für das Kind und von den Untersuchungen so effektiv wie möglich gestaltet werden.

\section{Literatur}

1 Arendt K, Demaerschalk BM, Wingerchuk DM et al. A traumatic lumbar puncture needles: After all these years, are we still missing the point? Neurology 2009; 15: 17-20

2 Bernbeck, B Wüller D, Janssen G et al. Symptoms of childhood acute lymphoblastic leukemia: red flags to recognize leukemia in daily practice. Klin Padiatr 2009; 221: 369-373

3 Brunner J, Moschovakis G, Prelog M. Lyme neuroborreliosis: etiology and diagnosis of facial palsy in children from tyrol. Klin Padiatr 2010; 222: 302-307

4 Christie LJ, Honarmand S, Perlkinton DF et al. Pediatric encephalitis: What is the role of myocoplasma pneumoniae? Pediatrics 2007; 120: 305-313

5 Ebinger F, Kosel C, Pietz J et al. Head ache and back ache after lumbar puncture in children and adolescent: A prospective study. Pediatrics 2004; 113: 1588-1592 
6 Fein D, Avner JR, Khine A. Pattern of pain management during lumbar puncture in children. Padiatr Emerg Care 2010; 26: 357-360

7 Glaser CA, Honarmand S, Anderson LJ et al. Beyond viruses: clinical profiles and etiologies associated with encephalitis. Clin Infect Dis 2006; 43: 1565-1577

8 Kimia AA, Kapraro AJ, Hummel D et al. Utility of lumbar puncture for first simple febrile seizures among children $6^{\text {th }}$ to $18^{\text {th }}$ month of age. Pediatrics 2009; 123: 6-12

9 Meyer S, Gortner L, Gottschling S. Cardiogenic shock in a neonate with enterovirus myocarditis. Klin Padiatr 2009; 221: 444-447
10 Shaked O, Pena BM, Linares MY et al. Simple febrile seizures: are the AAP guide lines regarding lumbar puncture being followed? Pediatr Emerg Care 2009; 25: 8-11

11 Völter C, Helms J, Weissbrich B et al. Frequent detection of myocoplasma pneumoniae in Bell's palsy. Eur Arch Otorhinolaryngol 2004; 261: 400-404

12 Yis U, Kurul SH, Cakmakci H et al. Myocoplasma pneumoniae: Nervous system complications in childhood and review of the literature. Eur J Pediatr 2008; 167: 973-978 